\title{
Boosting promotes advantageous risk-taking
}

Tomas Folke (i) ${ }^{1,2 \times}$, Giulia Bertoldo ${ }^{3}$, Darlene D'Souza ${ }^{4}$, Sonia Ali ${ }^{5}$, Federica Stablum (D) ${ }^{2}$ \& Kai Ruggeri (D) ${ }^{2,6}$

Due to the prevalence and importance of choices with uncertain outcomes, it is essential to establish what interventions improve risky decision-making, how they work, and for whom. Two types of low-intensity behavioural interventions are promising candidates: nudges and boosts. Nudges guide people to better decisions by altering how a choice is presented, without restricting any options or modifying the underlying payoff matrix. Boosts, on the other hand, teach people decision strategies that focus their attention on key aspects of the choice, which allows them to make more informed decisions. A recent study compared these two types of interventions and found that boosts worked better for risky choices aimed at maximising gains, whereas nudges worked best for choices aimed at minimising losses. Though intriguing, these findings could not be easily interpreted because of a limitation in the items used. Here we replicate that study, with an extended item set. We find that boosts work by promoting risk-taking when it is beneficial, whereas nudges have a consistent (lesser) impact, regardless of whether risk-taking is beneficial or not. These results suggest that researchers and policymakers should consider the base rate risk propensity of the target population when designing decision-support systems.

\footnotetext{
${ }^{1}$ Department of Mathematics and Computer Science, SASN, Rutgers University, Rutgers, NJ, USA. ${ }^{2}$ Centre for Business Research, Judge Business School, University of Cambridge, Cambridge, UK. ${ }^{3}$ School of Psychology, University of Padova, Padova, Italy. ${ }^{4}$ Department of Sociomedical Sciences, Columbia University, New York, USA. ${ }^{5}$ School of Psychology, University of Sussex, Sussex, UK. ${ }^{6}$ Department of Health Policy and Management, Columbia University, New York, USA. email: tomas.folke@gmail.com
} 


\section{Introduction}

isky decision-making is difficult, and people often do not make optimal choices when it comes to risk (Cox et al., 2015). Risky choices depend on risk preferences as well as the capacity for probabilistic reasoning, and there appears to be great variation in both (Dohmen et al., 2010; Hallahan et al., 2004; Grable, 2000). Despite the plenitude of interventions that have been designed to improve decision-making under risk, it is far from a solved problem.

One concept that has been central to formal reasoning about risky choices is expected value (EV). The EV of an option is the sum of its possible outcomes, weighted by their respective probabilities (Kagel and Roth, 2016). Picking the option with the highest EV is advantageous, in the sense that over infinite iterations of the same choice picking the highest EV option leads to the largest gains (or smallest losses). For this reason, picking the highest EV option will be referred to as "the advantageous choice" throughout this paper. This is intended as an imperfect shorthand rather than an absolute claim of optimality. Picking a low EV option can be self-consistent (e.g., when risk preferences overwhelm the wish to maximise rewards), and beneficial in specific contexts (e.g., when any loss would be catastrophic).

There have been numerous attempts in the behavioural sciences to help people manage risk better, however, such interventions do not always produce consistent effects (Hummel and Maedche, 2019), and sometimes fail to generalise from the context for which they are created (Mills, 2020). For example, it has long been concluded that individuals have different risk preferences for choices framed as maximising rewards rather than choices framed as minimising losses (Tversky and Kahneman, 1981). Whereas early work assumed that risk preferences for gains negatively predicted risk preferences for losses, contemporary studies have shown that risk preferences are independent between frames (Kurnianingsih and Mullette-Gillman, 2015; Tymula et al., 2013). This, in turn, may suggest that risktaking for gains and losses have different cognitive underpinnings, and thus are impacted differently by the same intervention. Some evidence in support of this possibility comes from Kurnianingsih and Mullette-Gillman, who found dissociations in how the value was encoded neurologically for gains and losses (Kurnianingsih and Mullette-Gillman, 2016). Though others have failed to detect a strict anatomical division between the encoding of gains and losses, these appear to activate different brain regions, with losses being more associated with amygdala activation, whereas gains are associated with activity in the ventromedial prefrontal cortex (vmPFC) (Pessiglione and Delgado, 2015). This distinction is further supported as patients with damaged amygdalas exhibit less loss aversion (De Martino et al., 2010). Finally, recent evidence suggests that manipulations of attentional load impact learning from prospective gains and losses differently, consistent with the idea that the effect of interventions may differ between these two domains (Boroujeni et al., 2021).

A recent study tested this concept by comparing the effectiveness of two common types of behavioural interventions: nudges and boosts (Franklin et al., 2019). Nudges involve small changes in the choice environment aiming to encourage better decisions without substantially altering the incentives of the options, often by changing how information is presented (Sunstein, 2014). Franklin et al. evaluated two nudge interventions: a disclosure nudge, which informed participants of the EV of each option, and a social nudge, that informed participants that the advantageous option was the most popular option.

Boosts support individuals to make more informed or wellconsidered choices by highlighting important features of the decision (Grüne-Yanoff and Hertwig, 2016; Hertwig, 2017; Hertwig and Grüne-Yanoff, 2021). These interventions have a successful track record in real-world applications, involving risky and uncertain choices both in the financial domain (Ruggeri et al., 2018), and adjacent domains such as household energy consumption (Heutel, 2019; Schleich et al., 2019). In experimental contexts, this typically involves simple, salient tools that assist participants in assigning clear valuations to choices. In the Franklin et al. experiment, for example, the boost intervention encouraged focused deliberation by teaching participants how to compute simple EVs and encouraged them to use this knowledge when making decisions. In more applied settings, boosts often take the form of calculators or rating tools/maps that allow decision-makers to input personal, critical information that is automatically converted into quantitative choices. For example, determining the ideal amount to pay on a credit card bill by determining available finances, income, the amount owed, and interest differences between savings and credit. These applications of boosts could potentially address economic inequalities given their focus on equity within interventions rather than applying one-size-fits-all choice suggestions.

In the Franklin et al. experiment, both the disclosure nudge and the boost increased the number of advantageous choices an individual made. However, the social nudge was ineffective, possibly because the participants did not view each other as a meaningful reference group for financial decisions. Additionally, Franklin et al. found that participants in the boost condition made the most advantageous choices for gain-framed items, whereas participants in the disclosure condition made the most advantageous choices for loss-framed items. In other words, the effectiveness of the interventions was moderated by the frame of the items, which is compatible with the theory that the cognitive structures that allow us to choose between risky options are different for gains and losses. Finally, Franklin et al. found that both nudges and boosts were most effective for those participants who picked suboptimal choices pre-intervention. They interpreted this to mean that the interventions were most beneficial for less competent decision-makers. This has important policy implications, because it may explain some of the heterogeneity in behavioural interventions (Bryan et al., 2021; Yarkoni, 2020) that may limit their effectiveness in public policy (Sunstein, 2017).

Franklin et al. provide insight into the effectiveness of nudges and boosts, yet the item set they used limited what conclusions could be drawn. For all experimental items in the gain frame the risky option was advantageous, whereas, for the experimental items in the loss frame, the certain option was advantageous. This conflation of item frame and risk advantage was intentional to reduce ceiling effects. However, it made it impossible to determine whether the boost intervention resulted in a greater propensity to pick the advantageous option, or if it just promoted risk-taking (which happened to be advantageous in this narrow experimental context). Additionally, the moderation effect between pre-intervention choices and the impact of the interventions can be interpreted in two equally valid ways. The authors interpreted this moderation from a skill perspective: participants who were unable to identify the highest EV option at baseline learned how to do so from the intervention. However, another explanation is equally consistent with the data: rather than teaching participants a new skill, the interventions improved the relative salience of EV relative to risk, so that the participants whose baseline risk-preferences were incompatible with maximising EV inhibited their risk preferences for the experimental task. Here we disambiguate these results by replicating Franklin et al. (2019) with an extended item set that orthogonalises item frame from risk advantage. Because the social nudge was ineffective in Franklin et al. (2019) we did not include that condition 
in this study, allowing us to obtain larger sample sizes for the remaining conditions.

This setup allows us to evaluate whether the interventions impact risk-taking differently depending on the frame of the choice. It also allows us to test whether the interventions specifically promote adaptive risk-taking or adaptive risk avoidance and whether their impact is moderated by underlying risk preferences. These distinctions are of societal importance because they would provide important information on how these interventions are best targeted, e.g., lack of savings has been associated with excessive risk-avoidance for gains (Carvalho et al., 2016), whereas people with gambling addiction are excessively riskseeking. In line with the recent literature, we expect that risk preferences will only be weakly correlated between frames, absent of intervention (Kurnianingsih and Mullette-Gillman, 2015; Tymula et al., 2013). We also expect both the disclosure nudge and boost intervention to increase the number of advantageous choices. We expect the frame of the items to moderate intervention effectiveness, but we are agnostic as to whether the interventions are particularly effective in promoting advantageous risk-seeking or advantageous risk avoidance. Finally, we expect that pre-intervention choices will interact with intervention effectiveness, but we are agnostic as to whether this effect will be best explained by baseline performance moderating the effectiveness of the interventions, or the interventions inhibiting risk preferences.

\section{Methods}

Stimuli. The experimental items were binary forced choices where one option involved a certain gain, and the other option involved some probability of a greater gain and some probability of no gain. Experimental items varied in terms of outcome magnitude, the probability of the uncertain outcome, risk advantage, and whether the choice was framed in terms of gains or losses. Each of these stimulus dimensions had two levels, leading to $2^{4}=16$ experimental items. For a full list of the items see Table 1 .

Procedure. Italian participants were recruited through the online recruitment platform Prolific and were paid the equivalent of 1.50 GBP for their participation. Prior to the survey, they gave informed consent. This study was granted IRB approval from the Centre for Business Research at Judge Business School, University of Cambridge. The survey began with three pre-intervention items intended to capture baseline risk preferences: two in the gain frame and one in the loss frame. One item in the gain frame and one item in the loss frame mimicked the structure of the experimental items. These were used to capture pre-intervention risk preferences. For the third item, the EVs for the risky and certain options were equal. This item was used to stratify participants so that the same proportion of risk-seeking individuals were assigned to each experimental condition (control, nudge, and boost), but was not included in any analyses.

In the control condition, participants were presented with the 16 experimental items immediately. In the nudge condition, participants read a brief explanation of EV and the EVs of the options were presented with the experimental items. For the boost intervention, EV was explained to participants and they were taught how to calculate EVs. They then had to compute the EV for three prospects and could not progress with the experiment until they had done so correctly. The experimental items were then presented in the same way as for the control group. For all conditions, the item order was randomised, as was the order of the response options. Once the experimental phase was completed, participants encountered an attention check that asked them about their favourite colour, but immediately afterwards they were instructed to write "tomato" into the text box. We modified the attention check relative to Franklin et al. (2019) to make it a "fair" attention check according to Prolific's guidelines (https://researcher-help.prolific.co/hc/en-gb/articles/ 360009223553-Using-attention-checks-as-a-measure-of-data-

quality). Finally, participants completed demographic questions pertaining to their gender, their age, country of residence, whether they were born in their country of residence or had migrated, level of education, relationship status, employment status, annual household income, and whether they used a calculator when answering the experimental items. For the full survey in English and Italian see Appendices A and B. For a graphic representation of the procedure see Fig. 1.

Participants. Four-hundred, twenty-seven participants completed the survey. Of these, one was excluded because their IP address had been used before, so we could not rule out that they had completed the survey previously. An additional 37 participants were dropped for failing the attention check (see the section "Procedure"), leading to a final sample of 389 . For a demographic overview (see Table 2).

Statistical models. The data from this experiment were analysed by hierarchical logistic regression models, with items nested within participants, and the $y$-variable encoding whether the advantageous (highest EV) option was chosen in a given trial. This formulation allows for the inclusion of item-level, participant-level, and condition-level effects in a single framework. We used this modelling approach to replicate the findings from Franklin et al. (2019), by only including the eight items the current study shares with that study (see Appendix C) and to evaluate the relative importance of propensity for risky choice vs. propensity for advantageous choice. The models are nested so that each new model is an extension of the preceding one.

Our null model assumes that participants vary in their propensity to pick the highest EV option, as a function of the frame of the item and whether the high-value option of that item was risky. In other words, we assume that our participants have an average tendency to maximise EV and an average tendency to be risk-seeking, but that these propensities are not captured by the pre-intervention items and are not meaningfully impacted by the interventions. We formalize these assumptions in the following hierarchical regression model:

$$
\begin{gathered}
\operatorname{Pr}\left(\text { Choice }_{i}=\text { EVmax }_{i}\right) \\
=\operatorname{logit}^{-1}\left(\begin{array}{c}
\alpha_{j[i]}+\beta_{1} \text { GainFrame }_{i}+\beta_{2} \text { RiskAdvantageous }_{i} \\
+\beta_{3} \text { GainFrame }_{i} \times \text { RiskAdvantageous }_{i}
\end{array}\right) \text { for } i=1, \ldots, I \\
\operatorname{logit}^{-1}(x)=\frac{e^{x}}{1+e^{x}} \\
\alpha_{j} \sim N\left(\mu_{\alpha}, \sigma_{\alpha}^{2}\right) \text { for } j=1, \ldots, J \\
\beta_{1,2,3} \sim N(0,5) \\
\mu_{\alpha} \sim N(0,5) \\
\sigma_{\alpha}^{2} \sim \operatorname{Exp}(1)
\end{gathered}
$$

GainFrame is coded 1 if the associated item was framed as a gain and 0 if it was framed as a loss. RiskAdvantageous is coded as 1 if the risky option was advantageous for the item and 0 if it was not. $\alpha j$ are participant-wise intercepts drawn from a normal distribution with weakly informative hyperparameters. I is the total number of trials (6832), whereas $J$ is the total number of participants (389).

Pre-intervention EV propensity: our second model represents the hypothesis that pre-intervention choices capture a propensity to make advantageous choices (and so predict post-intervention 


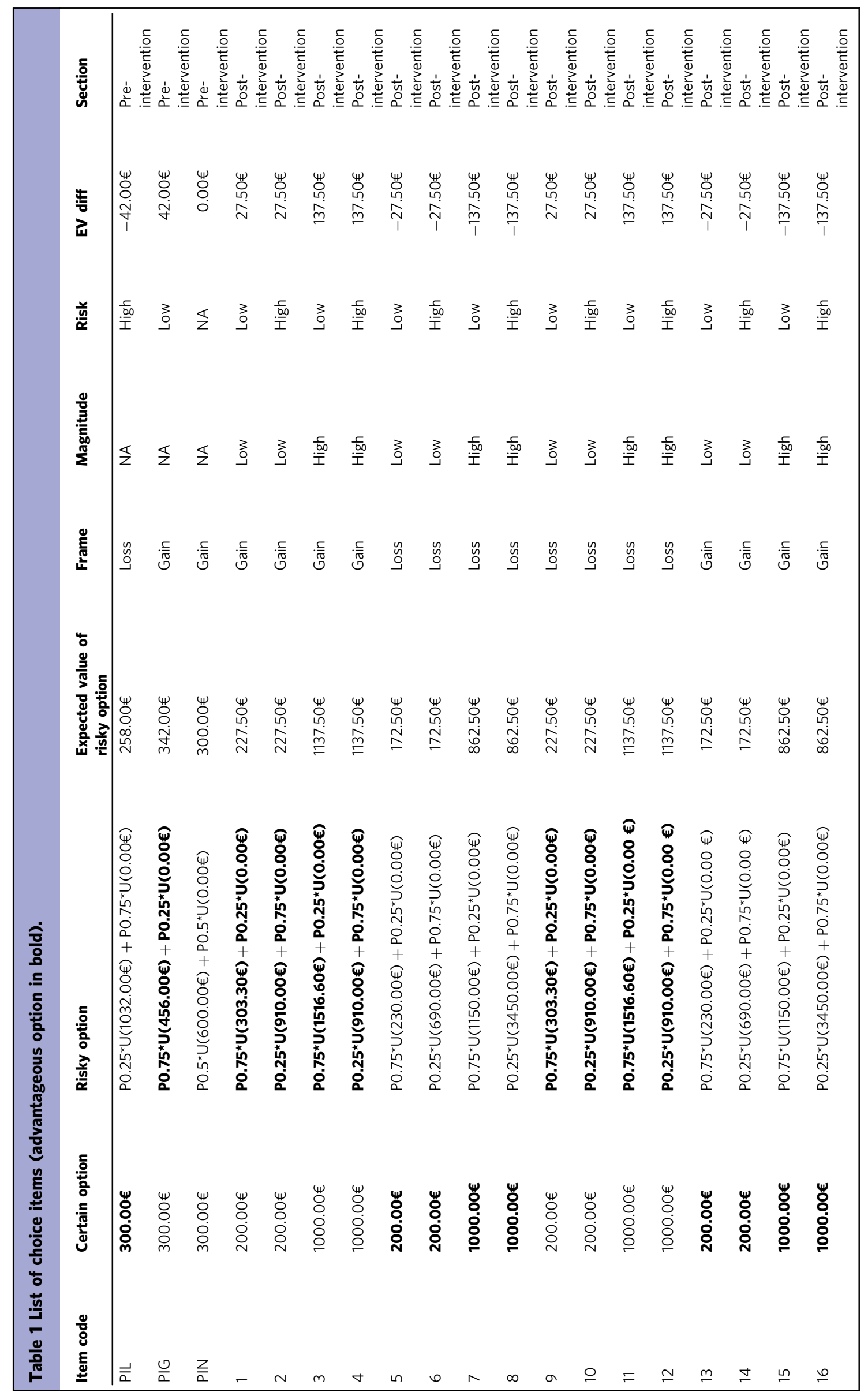




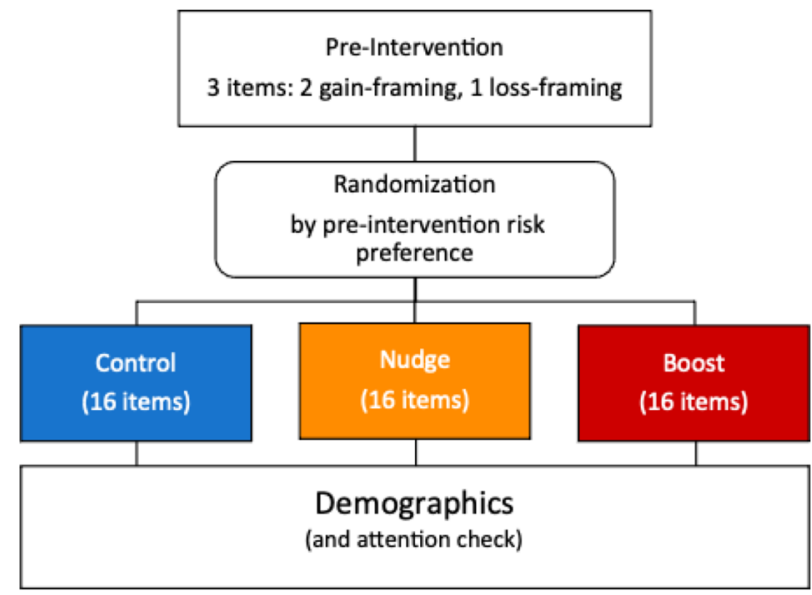

Fig. 1 The experimental procedure. Participants first completed three preintervention gambles, then were randomly assigned to one of three experimental conditions (stratified by baseline risk preference). Finally, all participants completed the same demographic section.

\begin{tabular}{|c|c|c|c|}
\hline $\begin{array}{l}\text { Gender } \\
\text { Male } \\
209(53.7 \%)\end{array}$ & $\begin{array}{l}\text { Female } \\
178(45.8 \%)\end{array}$ & $\begin{array}{l}\text { Other } \\
2(0.5 \%)\end{array}$ & \\
\hline $\begin{array}{l}\text { Age in years } \\
\text { Minimum }\end{array}$ & Median & Maximum & \\
\hline \\
\hline $\begin{array}{l}\text { Secondary School } \\
13(3.3 \%)\end{array}$ & $\begin{array}{l}\text { High School } \\
196(50.4 \%)\end{array}$ & $\begin{array}{l}\text { Undergraduate Degree } \\
101(26.0 \%)\end{array}$ & $\begin{array}{l}\text { Postgraduate Degree } \\
79(20.3 \%)\end{array}$ \\
\hline $\begin{array}{l}\text { Relationship status } \\
\text { Single } \\
158(40.6 \%)\end{array}$ & $\begin{array}{l}\text { In a relationship } \\
106(27.2 \%)\end{array}$ & $\begin{array}{l}\text { Engaged } \\
58(14.9 \%)\end{array}$ & $\begin{array}{l}\text { Married } \\
67(17.2 \%)\end{array}$ \\
\hline \multicolumn{4}{|c|}{ Annual household income in $1000 €$} \\
\hline 12 or less & $12-22$ & $22-31$ & 31 or more \\
\hline & $82(22.3 \%)$ & $90(24.5 \%)$ & $92(25.1 \%)$ \\
\hline
\end{tabular}

Table 3 Bayesian model comparisons.

\begin{tabular}{|c|c|c|c|c|}
\hline Model & WAIC & SE & $\triangle$ WAIC & $\Delta \mathrm{SE}$ \\
\hline 5. Moderation & 6859.7 & 76.6 & -3.7 & 8.7 \\
\hline 4. Intervention risk propensity & 6856.0 & 75.8 & 19.3 & 11.12 \\
\hline 3. Intervention EV propensity & 6875.3 & 75.6 & 29.5 & 12.0 \\
\hline 2. Pre-intervention risk propensity & 6904.8 & 74.6 & 360.2 & 43.8 \\
\hline 1. Pre-intervention EV propensity & 7265.0 & 68.1 & 42.8 & 15.8 \\
\hline O. Null & 7307.8 & 66.2 & & \\
\hline
\end{tabular}

choices), and that this propensity may differ by frame. We formalized this by expanding the null model so that:

$$
\begin{gathered}
\operatorname{Pr}\left(\text { Choice }_{i}=\text { EVmax }_{i}\right) \\
\ldots+\beta_{4} \text { PreIntGain }_{i}+\beta_{5} \text { PreIntLoss }_{i}+ \\
\beta_{6} \text { GainFrame }_{i} \times \text { PreIntGain }_{i}+ \\
\beta_{7} \text { GainFrame }_{i} \times \text { PreIntLoss }_{i} \\
\text { for } i=1, \ldots, I \\
\beta_{1, \ldots, 7} \sim N(0,5)
\end{gathered}
$$

PreIntGain was 1 if the participant picked the highest value option for pre-intervention gain frame item and 0 otherwise. PreIntLoss was 1 if the participant picked the highest value option for the pre-intervention loss frame item and 0 otherwise.
Pre-intervention risk propensity: our third model represents the hypothesis that pre-intervention items capture propensities for risky choice as well as advantageous choice. The intuition behind this model is that we included risk-advantage as a parameter so that our model could capture a pattern where participants were more likely to choose the high EV option when it was risky, but less likely to choose the high EV option when it was certain (or vice-versa). In other words, this more flexible model allowed for the possibility that the pre-intervention items captured participants' propensity to take risks, rather than their propensity to maximise EV. We extended model two so that:

$$
\begin{gathered}
\operatorname{Pr}\left(\text { Choice }_{i}=\text { EVmax }_{i}\right) \\
=\operatorname{logit}^{-1}\left(\begin{array}{c}
\ldots+\beta_{8} \text { PreIntGain }_{i} \times \text { RiskAdvantageous }_{i}+ \\
\beta_{9} \text { PreIntLoss }_{i} \times \text { RiskAdvantageous }_{i}+ \\
\beta_{10} \text { GainFrame }_{i} \times \text { RiskAdvantageous }_{i} \times \\
\text { PreIntGain }_{i}+\beta_{11} \text { GainFrame }_{i} \times \\
\text { RiskAdvantageous }_{i} \times \text { PreIntLoss }_{i} \\
\beta_{1, \ldots, 11} \sim N(0,5)
\end{array}\right) \text { for } i=1, \ldots, I
\end{gathered}
$$

Intervention EV propensity: our fourth model adds dummycoded fixed effects for the boost and nudges interventions, respectively, as well as interaction terms between the frame of the item and the intervention. It represents the hypothesis that the interventions increase advantageous choices independent of whether it is the risky or certain option that is advantageous.

$$
\begin{gathered}
\operatorname{Pr}\left(\text { Choice }_{i}=\text { EVmax }_{i}\right) \\
=\operatorname{logit}^{-1}\left(\begin{array}{c}
\cdots+\beta_{12} \text { Boost }_{i}+\beta_{13} \text { Nudge }_{i}+\beta_{14} \text { GainFrame }_{i} \\
\times \text { Boost }_{i}+\beta_{15} \text { GainFrame }_{i} \times \text { Nudge }_{i}
\end{array}\right) \text { for } i=1, \ldots, I \\
\beta_{1, \ldots, 15} \sim N(0,5)
\end{gathered}
$$

where Boost is coded as 1 if the experimental trial came from a participant in the boost condition and 0 otherwise, and Nudge is coded as 1 if the participant was in nudge condition and 0 otherwise.

Intervention risk propensity: our fifth model adds interaction terms between risk-advantage, intervention, and frame. Represents the hypothesis that interventions impact the propensity to select the highest EV option differently when that option is risky relative to when it is certain.

$$
=\operatorname{logit}^{-1}\left(\begin{array}{c}
\operatorname{Pr}\left(\text { Choice }_{i}=\text { EVmax }_{i}\right) \\
\ldots+\beta_{16} \text { Boost }_{i} \times \text { RiskAdvantageous }_{i}+\beta_{17} \text { Nudge }_{i} \\
\times \text { RiskAdvantageous }_{i}+\beta_{18} \text { GainFrame }_{i} \times \text { RiskAdvantageous }_{i} \\
\times \text { Boost }_{i}+\beta_{19} \text { GainFrame }_{i} \times \text { RiskAdvantageous }_{i} \times \text { Nudge }_{i}
\end{array}\right)
$$

Moderation: our sixth model adds fixed-effect interactions between the interventions, the experimental item frame, and preintervention choices. This model represents the hypothesis that the intervention effectiveness is moderated by pre-intervention 
choice.

$$
=\operatorname{logit}^{-1}\left(\begin{array}{c}
\operatorname{Pr}\left(\text { Choice }_{i}=\text { EVmax }_{i}\right) \\
\ldots+\beta_{20} \text { Boost }_{i} \times \text { PreIntGain }_{i} \times \\
\text { RiskAdvanatageous }_{i}+\beta_{21} \text { Boost }_{i} \times \text { PreIntLoss }_{i} \\
\times \text { RiskAdvanatageous }_{i}+\beta_{22} \text { Nudge }_{i} \times \text { PreIntGain }_{i} \times \\
\text { RiskAdvanatageous }_{i}+\beta_{23} \text { Nudge }_{i} \times \text { PreIntLoss }_{i} \times \\
\text { RiskAdvanatageous }_{i}+\beta_{24} \text { Boost }_{i} \times \text { PreIntGain }_{i} \times \\
\text { GainFrame }_{i} \times \text { RiskAdvantageous }_{i}+ \\
\beta_{25} \text { Boost }_{i} \times \text { PreIntLoss }_{i} \times \text { GainFrame }_{i} \times \\
\text { RiskAdvantageous }_{i}+\beta_{26} \text { Nudge }_{i} \times \text { PreIntGain }_{i} \\
\times \text { GainFrame }_{i} \times \text { RiskAdvantageous }_{i}+\beta_{27} \text { Nudge }_{i} \\
\times \text { PreIntLoss }_{i} \times \text { GainFrame }_{i} \times \text { RiskAdvantageous }_{i} \\
\text { for } i=1, \ldots, I \\
\beta_{1, \ldots, 27} \sim N(0,5)
\end{array}\right.
$$

We ran these models in rStan (Stan Development Team, 2018) on four chains with 12,000 samples per chain, the first 2000 in each discarded as warmup. All chains converged with Rhat values of 1 . We evaluated model performance based on the Widely Applicable Information Criterion (WAIC; Vehtari et al., 2017), as implemented in the rethinking R package (McElreath, 2016). As with other information criteria, lower WAIC scores indicate better performance. It is important to note that one should not compare "raw" WAIC scores and standard deviations in this case, because since these models share predictors, they also share information and estimation uncertainty, and we want to ignore this shared estimation uncertainty when comparing models (McElreath, 2016). This can be achieved by doing paired comparisons captured by the $\triangle \mathrm{WAIC}$, where positive numbers signify that more information is captured by the more complex model and negative numbers suggest that the simpler model best captures the data. In cases where the difference in WAIC is within the $\Delta \mathrm{SE}$, we can assume model equivalence, in which case we will favour the simpler model to maintain parsimony.

One benefit of Bayesian regression analysis is that one does not just come away with point estimates for regression coefficients, but rather full posterior distributions. This has the advantage that one naturally gets a range of effect sizes for each predictor within the model and that these ranges can be compared directly to determine the relative weights of the included predictors. An additional advantage of the Bayesian framework is that coefficients can be added together to produce posteriors for specific conditions. For example, if we want to know the null model log-likelihood of selecting the advantageous option for items in the gain frame where the risky option is advantageous, we can additively combine the posteriors of the intercept, the gain frame parameter, the risk advantage parameter, and the gain frame risk advantage interaction. This has the attractive property that it is easy to contrast theoretically important conditions that are encoded over multiple parameters.

We combined the posterior distributions of the coefficients of the best-fitting models in this way to reach a richer understanding of our results. For all comparisons between posterior distributions we report $p$, which is simply the proportion of posterior samples from the first distribution that are larger than the samples from the second distribution. This provides the strength of evidence of a directionality effect and can be read as "the probability that quantity 1 is greater than quantity 2 , conditional on the model assumptions and the data". To complement these directional probabilities, we also report total variation distance (TVD), which captures how similar or different two probability distributions are by evaluating their predictions. If the two distributions make the same predictions the TVD $=0$, and if they make completely nonoverlapping predictions TVD $=1$ (Gibbs and Su, 2002).

\section{Results}

Correlation between gains and losses. In line with previous work, we found that pre-intervention risk preferences are not significantly correlated across frames $(r=0.08,95 \% \mathrm{CI}=[-0.02-$ 0.18 ], $p=0.12$ ), and confidence intervals are bounded below 0.2 in either direction, so global effects are likely small. To further explore how consistent risk preferences are within and between frames, we grouped the experimental items based on frame (gain or loss) and risk advantage (whether the risky or certain option had the highest EV), and correlated risk preferences across these four categories for the control group. Risk preferences were fairly consistent across frames, but not across risk advantages. Specifically, items that shared the same frame but differed with regards to risk advantage correlated about twice as highly as items that shared the same risk advantage but differed with regards to frame (see Fig. 2).

Propensities for risk or EV: Model comparisons. As stated in the introduction, the main benefit of the current study is that it allows for the distinction between the propensity to pick risky options from the propensity to pick the advantageous option, because, in contrast to previous studies, the frame of each item is unrelated to whether the risky option is advantageous.

If pre-intervention choices capture propensity for advantageous choice rather than propensity for risk, they should predict advantageous choices regardless of whether the risky or certain option is advantageous. In contrast, if pre-intervention choices primarily capture risk propensities, they should be associated with more advantageous choices only when the risk advantage preand post-intervention match, and conversely, they should be associated with fewer advantageous choices when they are mismatched. These two hypotheses, pre-intervention choices capturing risk propensity vs pre-intervention choices capturing the propensity to maximise EV can be evaluated mathematically by including an interaction term between pre-intervention choice

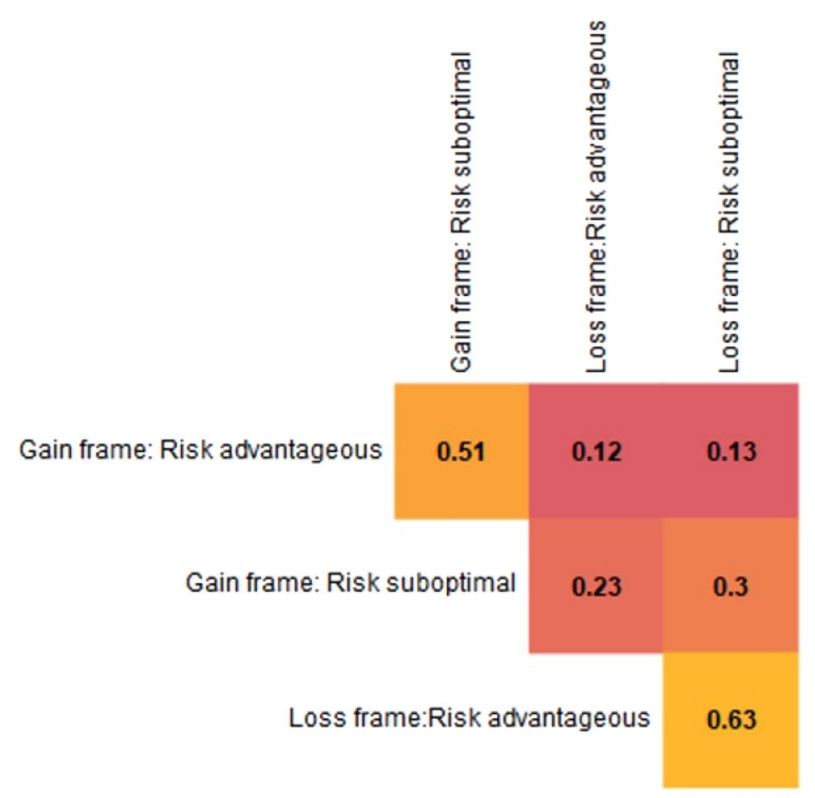

Fig. 2 Correlation between risky choices based on frame and risk advantage for the control group. Note. Items that share a frame but differ in risk advantage correlate more strongly than items that share the same advantageous option but differ on frame. 


\section{Frame of experimental item}

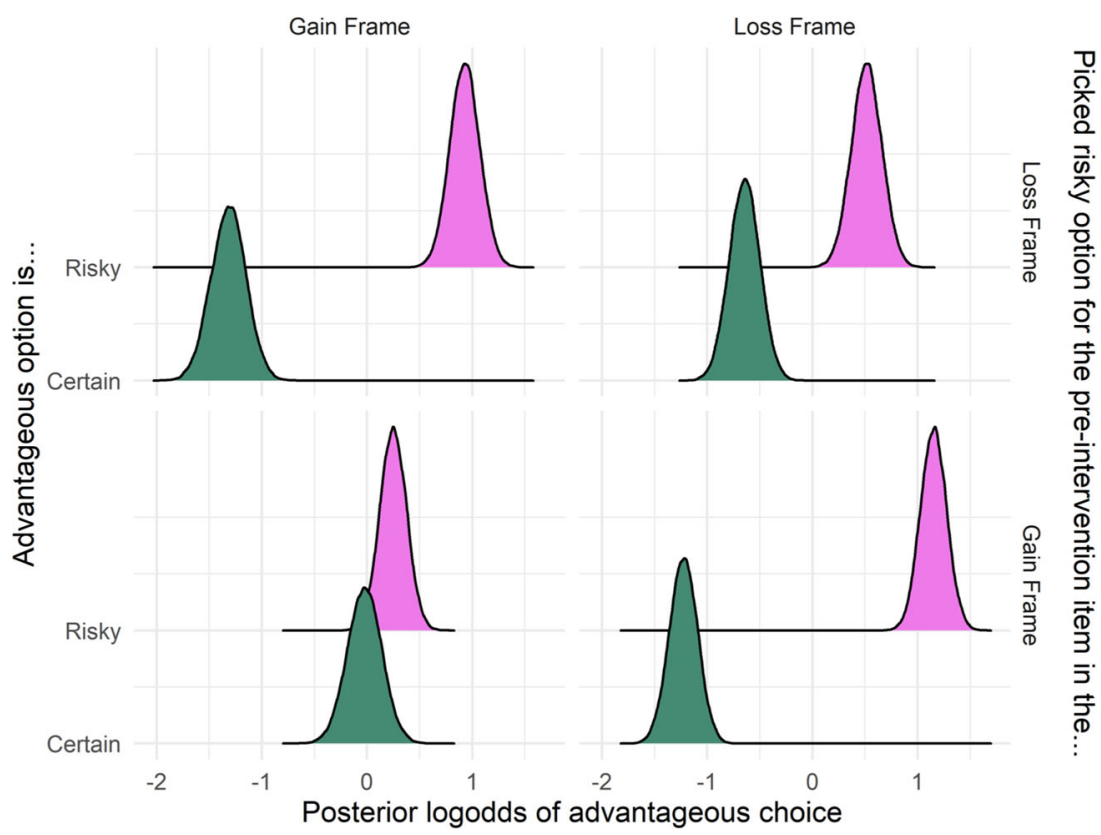

Fig. 3 Posterior log odds of advantageous choice as a function of risk. Posterior log odds of advantageous choice as a function of whether the preintervention choice was risky and whether the frame of the experimental item matched that of the pre-intervention item. Choosing the risky option preintervention is indicative of riskier choices post-intervention, regardless of whether the post-intervention frame is matching or not. In fact, pre-intervention choices are more predictive of risk preferences for experimental items with incongruent frames, as is illustrated by the fact that the distributions on the main diagonal are further apart than those on the counter diagonal.

and risk advantage. Such an interaction term should only improve model performance if the pre-intervention choice is a reliable indicator of risk propensity. The same logic can be applied to the interventions: if interventions impact risk propensities, interaction terms between the interventions and risk advantage should improve model performance, whereas if the interventions only impact the propensity to pick the advantageous option, they should not.

Note. Models are ordered in terms of complexity. Delta terms capture the difference between the current model and the model one row below, see Table 3 .

First, accounting for the impact of pre-intervention choices moderately improves model performance relative to the null model; accounting for whether the advantageous option was risky for the experimental items leads to a massive further improvement (nearly 8 times the standard error of the WAIC difference). This suggests that pre-intervention choices primarily capture risk preferences, as illustrated in Fig. 3. Accounting for experimental conditions led to further improvements in model performance, as did the extended model allowed the effect of the experimental conditions to vary based on risk advantage. This suggests that the interventions encourage certain advantageous choices and risky advantageous choices to a different extent. Finally, allowing preintervention choices to moderate the effectiveness of the experimental conditions lead to slightly worse model fit, indicating that the effectiveness of the interventions do not differ depending on pre-intervention choices. To summarise, these model comparisons suggest that pre-intervention choices primarily capture the propensity to take risks, rather than the propensity to choose high-EV options, and that the effectiveness of the interventions vary depending on whether the certain or risky option has the highest EV. To explore these effects more in detail we will analyse the posterior coefficients of the best fitting model (Model 4).
Propensities for risk or EV: Coefficient analyses. Figure 3 indicates that pre-intervention choices predict risk propensities, as it shows that participants who make risky choices prior to the intervention are more likely to pick the advantageous option post-intervention when it is risky, but are less likely to do so when it is certain. This pattern holds regardless of whether the preintervention frame matches the frame of the post-intervention item. Analytically, this is supported for the loss frame preintervention item both for experimental items in the loss frame $(p=1$, TVD $=0.99)$ and in the gain frame $(p=1$, TVD $=0.99)$. It is also true for the gain-frame pre-intervention item, regardless of whether the experimental item is in the loss frame $(p=1$, TVD $=0.99)$ or gain frame $(p=0.92$, TVD $=0.65)$. Surprisingly, the pre-intervention items seem to be more diagnostic of experimental risk preferences in the opposite frame both in the gain frame pre-intervention item $(p=1$, TVD $=0.99)$, and in the loss frame pre-intervention item $(p=0.99$, TVD $=0.99)$.

Finally, we explored the impact of the interventions on propensities for risk and EV (see Fig. 4). Participants made more advantageous choices when a certain option was advantageous $(p=1$, TVD $=1)$, signifying general risk-aversion. This gap between certain and risky advantageous choices was greater in the gain frame than in the loss frame $(p=1$, TVD $=1)$, signifying that the risk-aversion was stronger for gains than losses. In the gain frame, the boost condition was associated with more advantageous choices than the nudge condition $(p=1$, TVD $=0.99)$ and the control condition $(p=1$, TVD $=1)$. The same pattern held true for losses, with the boost intervention outperforming both the control condition $(p=1$, TVD $=1)$ and the nudge intervention $(p=1$, TVD $=0.99)$. There is strong evidence that the nudge condition outperformed the control condition in the gain frame $(p=0.99$, TVD $=0.92)$, and weaker evidence that it did so in the loss frame $(p=0.89$, TVD $=0.59)$. To summarise, the boost condition was associated with the most 


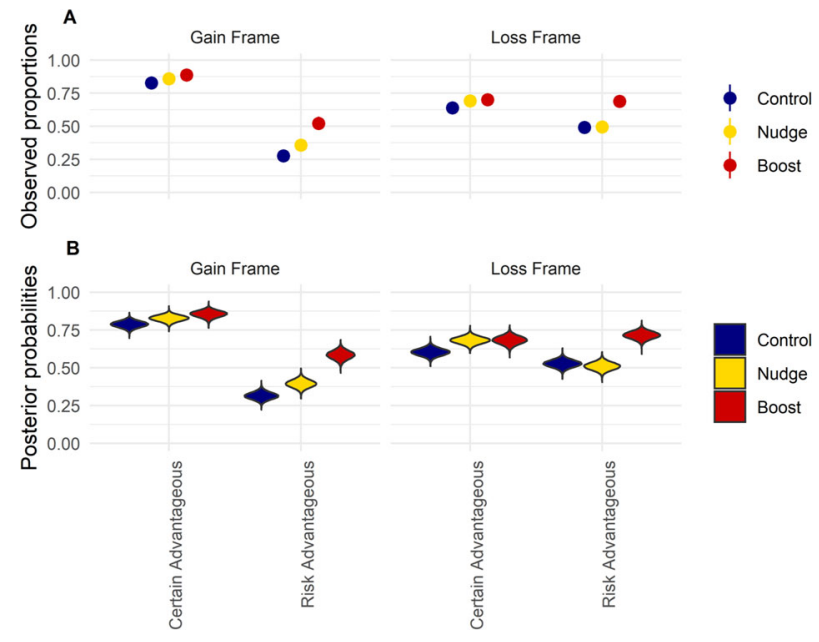

Fig. 4 Probability of advantageous choice as a function of item frame, risk advantage, and experimental condition. A Shows empirical means, error bars are bootstrapped $95 \% \mathrm{Cl}$. B Shows posterior probabilities from the best-fitting model (Model 4). The boost intervention is associated with more advantageous choices when the risky option is advantageous, for both gain frame and loss frame items. Intervention effects when the certain option is advantageous are much smaller.

advantageous choices for both frames, followed by the nudge condition, with the control condition being associated with the fewest advantageous choices. There is weak evidence that the effectiveness of the interventions in promoting advantageous choice varies by frame. Specifically, there is weak evidence that the boost intervention worked better in the gain frame than in the loss frame $(p=0.89, \mathrm{TVD}=0.54)$, and the same is true for the nudge intervention $(p=0.86, \mathrm{TVD}=0.47)$.

To investigate how the interventions impacted risk propensities, we compared the three experimental conditions based on item frame and risk advantage. For experimental items presented in the gain frame where the certain option was advantageous, we found that boosts were associated with a moderate increase in advantageous choices compared to the control condition $(p=0.99, \operatorname{TVD}=0.89)$ and the nudge condition $(p=0.85$, TVD $=0.51)$. Similarly, the nudge condition was associated with somewhat more advantageous choices than the control condition $(p=0.93, \mathrm{TVD}=0.68)$. When the risky option was advantageous, the boost intervention strongly dominated the nudge intervention $(p=1, \mathrm{TVD}=1)$ and the control condition $(p=1$, TVD $=1)$, and the nudge intervention was associated with reliably stronger performance than the control group $(p=0.99$, TVD $=0.90$ ). In the loss frame, we found that the boost condition and the nudge condition were associated with similar performances for items where a certain option was advantageous $(p=0.51, \mathrm{TVD}=0.07)$. Both interventions outperformed the control condition $\left(p_{\text {boost }}=0.99, \mathrm{TVD}_{\text {boost }}=0.89 ; p_{\text {nudge }}=0.99\right.$, $\left.\mathrm{TVD}_{\text {nudge }}=0.90\right)$. When the risky option was advantageous, the boost intervention outperformed both the nudge intervention $(p=1, \mathrm{TVD}=1)$ and the control condition $(p=1, \mathrm{TVD}=1)$, but the latter two groups had similar performances $(p=0.30$, TVD $=0.27$ ). These analyses suggest that the boost intervention led to more advantageous choices by encouraging risk-taking when it was advantageous to do so.

\section{Discussion}

This study explores how boosts and nudges influence risky decision-making; specifically, whether intervention effectiveness depends on how choices are framed, and whether risky or certain options are advantageous. We find that the boost intervention is associated with more advantageous choices than the nudge intervention, irrespective of choice frame. Furthermore, it seems that boosts work primarily by promoting risk-taking when risktaking is advantageous. This suggests a different interpretation of previously published findings, which found that the effectiveness of boosts depended on the chosen frame, but which conflated choice frame with whether risk-taking was advantageous (Franklin et al., 2019). These results have important real-world consequences because if boosts improve financial decisionmaking by promoting adaptive risk-taking, that implies that the effectiveness of boosts interventions depend on (1) the extent to which the environment rewards risk-taking and (2) the extent to which these opportunities are exploited. To put it plainly, boosts might lead to great improvements in environments that reward risk-taking and for populations who are excessively risk averse. This has societal implications because a field experiment found that people without savings are particularly risk averse for gains (Carvalho et al., 2016), which may serve as an additional barrier to wealth accumulation.

The other key insight from this work is that pre-intervention items seem to capture propensities for risk-seeking rather than propensities for high or low EVs. This finding complements the previous finding because it suggests that policymakers can identify people who would most benefit from boost interventions by studying their choice histories. Greater societal inequality has been associated with greater risk-taking (Payne et al., 2017), so interventions like ours might be particularly beneficial in those contexts. In contrast with previous work, we did not find that the informativeness of pre-intervention choices were higher for future choices with a matching frame. However, since we found that frame congruence did predict risk propensity for the control group, and pre-intervention choices were only captured by two items, these analyses may be underpowered. Future work should measure pre-intervention preferences more robustly to obtain a stronger sense of how they relate to framing effects and the extent to which they moderate the impact of interventions.

Finally, we found that both interventions were slightly more effective at promoting advantageous choice in the gain frame in the loss frame. This effect is tenuous and needs to be replicated in a larger sample. However, if it holds it would have important implications for real-world applications, as it implies that people are more sensitive to external circumstances when thinking about gains than when thinking about losses. Such findings have direct policy implications. The most evident is that behavioural interventions might be more effective when targeting savings, pensions and investments-which are unequivocally gain focused-than when targeting credit programs such as student loans and mortgages which has a loss component due to interest rates and the endowment effect associated with having to pay back what was given. These applications will be specifically relevant in addressing heterogeneity in choices created by economic inequalities (Adamkovič and Martončik, 2017).

The current research suffers from two primary limitations. First, the outcomes of the choices are hypothetical. However, earlier research suggests that participants tend to behave similarly for hypothetical and consequential decisions with regards to gambles and framing effects (Kühberger et al., 2002; Hoffmann et al., 2015; for a theoretical treatment see Read, 2005), and this pattern holds for this experimental setup (Franklin et al., 2019). Second, our results only cover a very specific type of risky choices, with binary options and a very simple pay-out structure. Realworld choices generally involve a much greater range of alternatives, that differ on a wider range of dimensions, and where both probabilities and pay-offs are less well-specified. This is particularly true once one considers nudge and boost applications outside of the domain of personal finances, e.g., healthcare (Patel 
et al., 2018) or energy justice (DellaValle and Sareen, 2020). Therefore, the current experimental task structure may overstate the benefit of the boost intervention, as the EV calculation is simple compared to most real-world settings. Additionally, it is worth noting that we have focused explicitly on monetary outcomes. This is intentional, because it is an outcome with attractive mathematical properties that most people have an intuitive understanding of, but it does raise questions about the generalisability of our results, as there is some evidence that risk attitudes vary depending on the type of outcome (Blais and Weber, 2006; see also Highhouse et al., 2017).

To address these limitations, future work should examine whether the general patterns outlined here apply to real-world choices. Specifically investigating how boosts can improve riskmanagement in relation to insurance, savings, and debt repayment.

\section{Conclusion}

Decision-making under risk is a ubiquitous problem, which is challenging to most people because they find probabilities counterintuitive. Teaching people about EVs is an easy way to help them reason more effectively about risky decisions when probabilities and outcomes are known. In this study, we compared two light touch interventions that do just that: a nudge that presents the EVs of each option and a boost that teaches participants how to calculate EVs themselves. We found that the boost was associated with picking the highest EV option more often than the nudge or a no-intervention control group, both for choices framed as gains and choices framed as losses, and that it operated by promoting adaptive risk-taking. It is an open question whether this pattern holds for real-world decisions that involve more complex prospects with multiple possible outcomes and less certain probabilities. Should these results prove to generalise, they have important implications for how to improve financial decision-making, especially for people with little training or experience in making such choices.

\section{Data availability}

The datasets, analysis code, and Bayesian models referenced in this study are available on the OSF: https://osf.io/9np3c/.

Received: 19 May 2021; Accepted: 11 October 2021;

Published online: 16 November 2021

\section{References}

Adamkovič M, Martončik M (2017) A review of consequences of poverty on economic decision-making: a hypothesized model of a cognitive mechanism. Front Psychol 8:1784

Blais AR, Weber EU (2006) A domain-specific risk-taking (DOSPERT) scale for adult populations. Judgm Decision Mak 1:1

Boroujeni KB, Watson M, Womelsdorf T (2021) Gains and losses affect learning differentially at low and high attentional load. Preprint at https://doi.org/ $10.1101 / 2020.09 .01 .278168$

Bryan CJ, Tipton E, Yeager DS (2021) Behavioural science is unlikely to change the world without a heterogeneity revolution. Nat Hum Behav 5(8):980-989

Carvalho LS, Prina S, Sydnor J (2016) The effect of saving on risk attitudes and intertemporal choices. J Dev Econ 120:41-52

Cox JC, Sadiraj V, Schmidt U (2015) Paradoxes and mechanisms for choice under risk. Exp Econ 18(2):215-250. https://doi.org/10.1007/s10683-014-9398-8

DellaValle N, Sareen S (2020) Nudging and boosting for equity? Towards a behavioural economics of energy justice. Energy Res Soc Sci 68:101589

De Martino B, Camerer CF, Adolphs R (2010) Amygdala damage eliminates monetary loss aversion. Proc Natl Acad Sci USA 107(8):3788-3792

Dohmen T, Falk A, Huffman D, Sunde U (2010) Are risk aversion and impatience related to cognitive ability? Am Econ Rev 100(3):1238-1260. https://doi.org/ 10.1257/aer.100.3.1238
Franklin M, Folke T, Ruggeri K (2019) Optimising nudges and boosts for financia decisions under uncertainty. Palgrave Commun 5(1):113. https://doi.org/ 10.1057/s41599-019-0321-y

Gibbs AL, Su FE (2002) On choosing and bounding probability metrics. Int Stat Rev 70(3):419-435

Grable JE (2000) Financial risk tolerance and additional factors that affect risk taking in everyday money matters. J Bus Psychol 14(4):625-630. https:// doi.org/10.1023/A:1022994314982

Grüne-Yanoff T, Hertwig R (2016) Nudge versus boost: how coherent are policy and theory? Minds Mach 26(1):149-183. https://doi.org/10.1007/s11023-0159367-9

Hallahan T, Faff R, Mckenzie M (2004) An empirical investigation of personal financial risk tolerance. Financ Serv Rev 13(1):57-78

Hertwig R (2017) When to consider boosting: some rules for policy-makers. Behav Public Policy 1(2):143-161. https://doi.org/10.1017/bpp.2016.14

Hertwig R, Grüne-Yanoff T (2021) Boosting and nudging: two paths toward better financial decisions. In: Viale R, Filotto U, Alemanni B, Mousavi S (ed) Financial education and risk literacy. Edward Elgar Publishing

Heutel G (2019) Prospect theory and energy efficiency. J Environ Econ Manag 96:236-254

Highhouse S, Nye CD, Zhang DC, Rada TB (2017) Structure of the Dospert: is there evidence for a general risk factor? J Behav Decision Mak 30(2):400-406

Hoffmann AO, Post T, Pennings JM (2015) How investor perceptions drive actual trading and risk-taking behavior. J Behav Financ 16(1):94-103

Hummel D, Maedche A (2019) How effective is nudging? A quantitative review on the effect sizes and limits of empirical nudging studies. J Behav Exp Econ 80:47-58. https://doi.org/10.1016/j.socec.2019.03.005

Kagel JH, Roth AE (eds) (2016) The handbook of experimental economics, vol 2. Princeton University Press

Kühberger A, Schulte-Mecklenbeck M, Perner J (2002) Framing decisions: hypothetical and real. Organ Behav Hum Decision Process 89(2):1162-1175

Kurnianingsih YA, Mullette-Gillman OA (2015) Divergence and convergence of risky decision making across prospective gains and losses: preferences and strategies. Front Neurosci 9 https://doi.org/10.3389/fnins.2015.00457

Kurnianingsih YA, Mullette-Gillman OA (2016) Neural mechanisms of the transformation from objective value to subjective utility: converting from count to worth. Front Neurosci 10:507

McElreath R (2016) rethinking: Statistical rethinking book package. R package version 1 . Taylor \& Francis

Mills S (2020) Personalized nudging. Behav Public Policy 1-10. https://doi.org/ $10.1017 / \mathrm{bpp} .2020 .7$

Patel MS, Volpp KG, Asch DA (2018) Nudge units to improve the delivery of health care. New Engl J Med 378(3):214

Payne BK, Brown-Iannuzzi JL, Hannay JW (2017) Economic inequality increases risk taking. Proc Natl Acad Sci USA114(18):4643-4648

Pessiglione M, Delgado MR (2015) The good, the bad and the brain: neural correlates of appetitive and aversive values underlying decision making. Curr Opin Behav Sci 5:78-84

Read D (2005) Monetary incentives, what are they good for? J Econ Methodol 12(2):265-276

Ruggeri K, Folke T, Jarke H, Paul A, Gladstone FJ (2018) Economic, financial and consumer decision-making. In: Ruggeri $\mathrm{K}$ (ed) Behavioural insights for policy: concepts and cases. Routledge, pp. 156-179

Schleich J, Gassmann X, Meissner T, Faure C (2019) A large-scale test of the effects of time discounting, risk aversion, loss aversion, and present bias on household adoption of energy-efficient technologies. Energy Econ 80:377-393

Stan Development Team (2018) RStan: The R interface to Stan. R package version 2.17.3. Stan Development Team http://mc-stan.org

Sunstein CR (2014) Nudging: a very short guide. J Consum Policy 37(4):583-588

Sunstein CR (2017) Nudges that fail. Behav Public Policy 1(1):4-25

Tversky A, Kahneman D (1981) The framing of decisions and the psychology of choice. Science 211(4481):453-458. https://doi.org/10.1126/science.7455683

Tymula A, Rosenberg Belmaker LA, Ruderman L, Glimcher PW, Levy I (2013) Like cognitive function, decision making across the life span shows profound age-related changes. Proc Natl Acad Sci USA 110(42):17143-17148. https:// doi.org/10.1073/pnas.1309909110

Vehtari A, Gelman A, Gabry J (2017) Practical Bayesian model evaluation using leave-one-out cross-validation and WAIC. Stat Comput 27(5):1413-1432. https://doi.org/10.1007/s11222-016-9696-4

Yarkoni T (2020) The generalizability crisis. Behav Brain Sci 1-37. https:// www.cambridge.org/core/journals/behavioral-and-brain-sciences/article/abs/ generalizability-crisis/AD386115BA539A759ACB3093760F4824

\section{Acknowledgements}

This study was funded in part by the Economic and Social Research Council, Global Challenges Research Fund (ES/P010962/1) within the R4HC-MENA project. 


\section{Competing interests}

The authors declare no competing interests.

\section{Ethical approval}

This article does not contain any studies with human participants performed by any of the authors.

\section{Informed consent}

This article does not contain any studies with human participants performed by any of the authors.

\section{Additional information}

Supplementary information The online version contains supplementary material available at https://doi.org/10.1057/s41599-021-00942-3.

Correspondence and requests for materials should be addressed to Tomas Folke.

Reprints and permission information is available at http://www.nature.com/reprints
Publisher's note Springer Nature remains neutral with regard to jurisdictional claims in published maps and institutional affiliations.

(c) (i) Open Access This article is licensed under a Creative Commons Attribution 4.0 International License, which permits use, sharing, adaptation, distribution and reproduction in any medium or format, as long as you give appropriate credit to the original author(s) and the source, provide a link to the Creative Commons license, and indicate if changes were made. The images or other third party material in this article are included in the article's Creative Commons license, unless indicated otherwise in a credit line to the material. If material is not included in the article's Creative Commons license and your intended use is not permitted by statutory regulation or exceeds the permitted use, you will need to obtain permission directly from the copyright holder. To view a copy of this license, visit http://creativecommons.org/ licenses/by/4.0/.

(C) The Author(s) 2021 